concentrischen ringförmigen Flammen. Ibr Vortheil soll in der erzeugten hohen Temperatur und in der guten Vertheilung derselben bestehen, welche das Springen der damit erhitzten Gefässe möglichst verhindert. Die Aussteller dieser Lampen, die HH. Rohrbeck \& Co. in Berlin, hoffen, den jetzt noch hohen Preis derselben wesentlich reduciren zu können.

Für die Bibliothek sind eingegangen:

Classen, Alexander: Grundriss der Analytischen Chemie. I. Theil: Qualitative Analyse. Vom. Verf.

Ferner folgende Zeitschriften im Austausch:

Archives des sciences phys. et nat. (Genève). Novembre.

Annalen der Chemie und Pharm. Bd. 170. Heft 1, 2.

Deutsche Industriezeitung. No. 48, 49.

Annalen der Landwirthschaft. No. 94-97.

Revue hebdomadaire. No. 43.

Chemisches Centralblatt. No. 48, 49 .

Revue scientifique. No. $22,23$.

Bulletin de l'Académie royale de Belgique. No. 9, 10.

Verhandlungen der K. K. geolog. Reichsanstalt zu Wien. No. 14.

Bericht des Offenbacher Vereins für Naturkunde. No. 13.

Bulletin de la Société chimique de Paris. Decembre.

\title{
Durch Kauf:
}

Comptes rendus. No. 18, 19, 20.

Polytechnisches Journal von Dingler. Bd. 210. Heft 4.

\section{Mittheilungen.}

\section{Julius Thomsen: Fortsetzung der Untersuchung über einige 0xydations- und Reductionsmittel.}

(Eingegangen am 4. December.)

In diesen Berichten VI, S. 233 habe ich die Hauptresultate einer Untersuchung über einige Oxydations- und Reductionsmittel mitgetheilt. Spätere Untersuchungen haben mir aber gezeigt, dass bei der Bestimmung der unterchlorigen Säure ein Fehler sich eingeschlichen batte, und da diese Säure zur Bestimmung mehrerer der untersuchten Reactionen benutat worden war und demnach die Resultate ungenau gemacht hatte, entschloss ich mich, die ganze Untersucbung von vorn an wieder durchzuführen, und zwar in der Art, dass nun kein Zweifel über die Zuverlässigkeit der Resultate stattfinden kann. Ich habe nämlich jetzt sämmtliche Werthe nach mehreren, unter einander unabbängigen Methoden bestimmt und übereinstimmende Resultate erreicht.

Hr. Ber thelot, der wahrseheinlicb mit ähnlichen Untersuchungen beschäftigt gewesen ist, hat im Bull. soc. chim. $X X, 111$ einen Augriff 
auf meine Methode gerichtet. Es scheint aber, dass Hr. Berthelot meine Formeln nicht verstanden hat; denn erstens spricht er stark gegen die Anwendung von Chlorwasser als Oxydationsmittel bei thermo-chemischen Messungen, obgleich keine meiner Bestimmungen mittelst Chlorwasser ausgeführt ist, und ferner zeigt er, dass die Wärme bei der Reaction von gasförmigem Chlor auf Zinnchlorürlösung mit dem Säuregehalt der Lösnng variirt, was ebenfalls auf meine Bestimmungen keinen Einfluss hat, da ich die Grösse durch die Reaction von unterchloriger Säure auf Zinnchlorür bestimmt hatte.

Ich muss mich bier auf eine Angabe der Hauptresultate meiner neven Untersuchung beschränken. Die Abbandlung selbst, welche an Poggendorff's Annalen eingesandt ist, enthält alle Einzelheiten der Untersuchung, bezüglich welcher ich auf dieselbe verweise.

1) Die unterchlorige $S$ äure babe ich nach $z$ wei Methoden untersucht, von welchen die eine der Bildung, die andere der Zersetzung der Säure entspricht. Die untersuchten Reactionen sind:

a) Absorption von Chlor durch Natronlösung.

b) Reduction der unterchlorigen Säure durch Jodwasserstoff.

Diese beiden Methoden haben folgende Resultate gegeben:

$$
(\mathrm{Cl}, \mathrm{O}, \mathrm{H}, \mathrm{Aq})=\left\{\begin{array}{c}
\mathrm{a} \ldots 29963^{\mathrm{c}} \\
\mathrm{b} \ldots 29793 \\
\text { Mittel 29878c }
\end{array}\right.
$$

Diese Werthe stützen sich nur auf Kenntniss der Bildungswärme des Wassers, der Chlor- und der Jodwasserstoffsäure und der Neutralisationswärme des Natrons.

2) Die Jodsäure ist ebenfalls nach zwei Methoden untersucht: einerseits wurde die Säure in ihre Bestandtheile zersetzt, andererseits die Bildung der Säure aus ibren Bestandtbeilen vollzogen. Die benutzten Reactionen waren:

a) Reduction von Jodsäure durch Jodwasserstoff.

b) Oxydation von Jodwasserstoff durch unterchlorige Säure.

Die Resultate nach diesen beiden Methoden sind:

$$
\left(\mathrm{J}, \mathrm{O}^{3}, \mathrm{H}, \mathrm{Aq}\right)=\left\{\begin{array}{c}
\mathrm{a} \ldots 55844^{\mathrm{e}} \\
\mathrm{b} \ldots 55542 \\
\text { Mittel } 55713^{\mathrm{c}} .
\end{array}\right.
$$

Da diese Werthe sich nur auf Wasser, unterchlorige Säure und Chlor- und Jodwasserstoff stützen, bilden sie gleichzeitig eine Controle für die diesen Körpern beigelegten Werthe.

3) Oxydationswärme des Zinnchlorürs. Diesen Werth habe ich auf dreifache Art bestimmt, von welchen die eine Bestimmung den Werth ganz unabhängig von allen bekannten Grössen giebt, sodass die Berechnung nicht einmal Kenntniss der calorischen 
Constanten des Wassers und des Chlorwasserstoffs voraussetzt. Die Oxydationswärme ist bestimmt worden:

a) mittelst unterchloriger Säure,

b) mittelsı Jodsäure,

c) durch die Kreismethode.

Für unseren speciellen Fall ist die Kreismethode die folgende.

Drei Lösungen: übermangansaures Kali, chlorwasserstoffhaltiges Zinnchlorür und chlorwasserstoff haltiges Wasserstoffhyperoxyd, lässt man zu zwei und zwei auf einander reagiren. Es sei die Wärmetönung bei der Reaction von $1 \mathrm{Mol}$. Zinnchlorür auf äquivalente Mengen von übermangansaurem Kali und Wasserstoffhyperoxyd bezüglich $A$ und B und die Wärmetönung bei der Reaction von Wasserstoffhyperoxyd auf übermangansaures Kali $\mathrm{C}$, dann ist

$$
\mathrm{A}+\mathrm{B}-\mathrm{C}=2 \mathrm{R} \text {, }
$$

wenn $R$ die Oxydationswärme des Zinnchlorürs bezeichnet. Es stützt sich demnach diese Bestimmung von $R$ auf keine anderen Beobachtungen. Die Resultate dieser drei Methoden sind folgende:

$$
\left(\mathrm{Sn} \mathrm{Cl}{ }^{6} \mathrm{H}^{4} \mathrm{Aq}, \mathrm{O}\right)=\left\{\begin{array}{c}
\mathrm{a} \ldots 65611^{\mathrm{c}} \\
\mathrm{b} \ldots 66010 \\
\mathrm{e} \ldots 65617 \\
\text { Mittel } 65746^{\mathrm{c}} .
\end{array}\right.
$$

Addiren $\operatorname{wir}^{-} \mathbf{z u}$ dieser Grösse den Werth

so resultirt

$$
2(\mathrm{H}, \mathrm{Cl}, \mathrm{Aq})-\left(\mathrm{H}^{2}, \mathrm{O}\right)=10273^{\circ},
$$

$$
\left(\mathrm{Sn} \mathrm{Cl}^{6} \mathrm{H}^{4} \mathrm{Aq}, \mathrm{Cl}^{2}\right)=76019 \text { ? }
$$

als die Wärmetönung, welche der Bildung von Zinnchlorid mittelst einer chlorwasserstoffhaltigen Lösung von Zinnchlorür und gasförmigem Chlor entspricht. Hr. Berthelot giebt den Werth $78600^{e}$. Ich glaube aber, dass er sich bezüglich der specifischen Wärme dieser viel Chlorwasserstoff enthaltenden Lösungen geirrt hat; denn das calorimetrische Aequivalent solcher Lösungen ist stets bedeutend geringer, als das in denselben enthaltene Wasser.

4) Das übermangansaure Kali als Oxydationsmittel. Die Wärmetönung bei der Zersetzung dieses Salzes in chlorwasserstoffsaurer Lösung unter Abgabe von 5 Atomen Sauerstoff resultirt:

a) aus der Reduction mittelst Zinnchlorür,

b) aus der Reduction mittelst Wasserstoffhyperoxyd.

Es geben diese beiden Processe folgende Werthe:

$$
\frac{1}{5}\left(\mathrm{Mn}^{2} \mathrm{O}^{8} \mathrm{~K}^{2} \mathrm{Aq}, 6 \mathrm{HCl} \mathrm{Aq}\right)=\left\{\begin{array}{l}
\text { a } \ldots 11599^{\circ} \\
\mathrm{b} \ldots 11856 \\
\text { Mittel 11727 }
\end{array}\right.
$$


Die Wärmeentwickelung. bei der Bildung von Manganhyperoxyd aus Manganoxydulhydrat und Sauerstoff ist aus folgenden $z$ wei Processen bestimmt:

a) Oxydation des Mangansulfats mittelst übermangansauren Kalis.

b) Reduction des Manganhyperoxydhydrats mittelst Eisensulfats.

Im ersten Processe wird Manganhyperoxyd gebildet, im zweiten wieder zersetzt. Die Resultate sind:

$$
(\overline{\overline{\mathrm{Mn}}}, \mathrm{O})=\left\{\begin{array}{l}
\mathrm{a} \ldots 21249^{\mathrm{c}} \\
\mathrm{b} \ldots 21774 \\
\text { Mittel } 21511^{\circ}
\end{array}\right.
$$

Aus diesen Grössen wird nun die Wärmetönung für andere Zersetzungen der Manganoxyde berechnet; ich habe die Resultate in der am Schluss beigefügten Tafel zusammengestellt.

5) Die Oxydationswärme des Eisenchlorürs. Meine Eisenlösung hatte die Zusammensetzung $\mathrm{Fe} \mathrm{Cl}^{2}+2 \mathrm{H} \mathrm{Cl}$, enthielt demnach pro Molekül $2 \mathrm{Mol}$. Chlorwasserstoff. Die Oxydationswärme derselben wurde nach drei Methoden bestimmt:

a) mittelst unterchloriger Säure,

b) mittelst übermangansauren Kalis,

c) mittelst gasförmigen Chlors.

Die Resultate sind die folgenden:

$$
\left(\mathrm{Fe}^{2} \mathrm{Cl}^{8} \mathrm{H}^{4} \mathrm{Aq}, \mathrm{O}\right)=\left\{\begin{array}{c}
\mathrm{a} \ldots 45556^{\mathrm{c}} \\
\mathrm{b} \ldots 44988 \\
\mathrm{c} \ldots 45207 \\
\text { Mittel } 45250^{\mathrm{c}} .
\end{array}\right.
$$

Addiren wir hierzu den Werth

dann resultirt

$$
2(\mathrm{Cl}, \mathrm{H}, \mathrm{Aq})-\left(\mathrm{H}^{2}, \mathrm{O}\right)=10273^{\mathrm{c}},
$$

$$
\left(\mathrm{Fe}^{2} \mathrm{Cl}^{8} \mathrm{H}^{4} \mathrm{Aq}, \mathrm{Cl}^{2}\right)=55523^{\mathrm{c}}
$$

für die Reaction von gasförmigem Chlor auf Eisenchlorürlösung.

6) Die Reactionsconstanten der Reductions- und Oxydationsmittel, sowie sie nach diesen Angaben sich herausstellen, und wie ich sie als zuverlässig bestimmt betrachte, habe ich in folgenden Tafeln zusammengestellt, die demnach an die Stelle der entsprechenden Tafeln meiner Abbandlung in diesen Berichten VI, S. 233 treten müssen. 
Tafel II. Reactionsconstanten der Reductionsmittel.

\begin{tabular}{|c|c|c|}
\hline Der Process & $Q$ & \\
\hline $\begin{array}{l}\left(\mathrm{SO}^{2} \mathrm{Aq}, \mathrm{Cl}^{2}\right) \ldots \ldots \\
\left(\mathrm{Fe}^{2} \mathrm{Cl}^{8} \mathrm{H}^{4} \mathrm{Aq}, \mathrm{Cl}^{2}\right) \ldots \\
\left(2 \mathrm{Fe} \mathrm{SO}^{4} \mathrm{Aq}, \mathrm{Cl}^{2}\right) \ldots \\
\left(\mathrm{Sn}^{6} \mathrm{Cl}^{6} \mathrm{H}^{4} \mathrm{Aq}, \mathrm{Cl}^{2}\right) \ldots \\
\left(\mathrm{SO}^{2} \mathrm{Aq}, \mathrm{O}\right) \ldots \\
\left(\mathrm{Fe}^{2} \mathrm{Cl}^{8} \mathrm{H}^{4} \mathrm{Aq}, \mathrm{O}\right) \ldots \\
\left(2 \mathrm{Fe} \mathrm{SO}^{4} \mathrm{Aq}, \mathrm{O}\right) \ldots \\
\left(\mathrm{Sn} \mathrm{Cl}^{6} \mathrm{H}^{4} \mathrm{Aq}, \mathrm{O}\right) \ldots\end{array}$ & $\left.\begin{array}{l}73907 \mathbf{5} \\
55523 \\
48763 \\
76019 \\
63634 \\
45250 \\
38490 \\
65746\end{array}\right)$ & für 1 Atom Sauerstoff. \\
\hline
\end{tabular}

Tafel III. Reactionsconstanten der Oxydationsmittel.

\begin{tabular}{|c|c|c|c|}
\hline Reaction & $Q^{\prime}$ & $\begin{array}{c}\text { Disponible } \\
\text { Sauer- } \\
\text { stoffatome }\end{array}$ & $\begin{array}{c}\mathbf{Q} \\
\text { pro } 1 \text { Atom } \\
\text { Sauerstoff }\end{array}$ \\
\hline $2(\mathrm{Cl}, \mathrm{H}, \mathrm{Aq})-\left(\mathrm{H}^{2}, \mathrm{O}\right)$ & $10273^{\mathrm{c}}$ & 1 & 10273 \\
\hline $2(\mathrm{Br}, \mathrm{H}, \mathrm{Aq})-\left(\mathrm{H}^{2}, \mathrm{O}\right) \ldots$ & -11605 & 1 & -11605 \\
\hline $2(\mathrm{Br} A \mathrm{q}, \mathrm{H})-\left(\mathrm{H}^{2}, \mathrm{O}\right)$ & -12683 & 1 & -12683 \\
\hline$(\mathrm{Cl}, \mathrm{H}, \mathrm{Aq})-(\mathrm{Cl}, \mathrm{O}, \mathrm{H}, \mathrm{Aq}) \ldots$ & 9437 & 1 & 9437 \\
\hline$\left(\mathrm{Mn}^{2} \mathrm{O}^{8} \mathrm{~K}^{2} \mathrm{Aq}, 6 \mathrm{H} \mathrm{Cl} \mathrm{Aq}\right) \ldots$ & 58635 & 5 & 11727 \\
\hline$\left(\mathrm{Mn}^{2} \mathrm{O}^{8} \mathrm{~K}^{2} \mathrm{Aq}, 3 \mathrm{SO}^{3} \mathrm{Aq}\right) \ldots$ & 69485 & 5 & 13897 \\
\hline$\left(\mathrm{Mn}^{2} \mathrm{O}^{8} \mathrm{~K}^{2} \mathrm{Aq}, 2 \mathrm{H} \mathrm{Cl} \mathrm{Aq}\right) \ldots$ & 55757 & 3 & 18586 \\
\hline$\left(\mathrm{Mn}^{2} \mathrm{O}^{8} \mathrm{~K}^{2} \mathrm{Aq}, \mathrm{SO}^{3} \mathrm{Aq}\right) \ldots$ & 59547 & 3 & 19849 \\
\hline$\left(\mathrm{MnO} O \mathrm{SO}^{3} \mathrm{Aq}\right) \ldots \ldots$ & 4969 & 1 & 4969 \\
\hline$\left(2 \mathrm{CrO}^{3} \mathrm{Aq}, 3 \mathrm{SO}^{3} \mathrm{Aq}\right)$ & 30452 & 3 & 10151 \\
\hline$-\left(2 \mathrm{Mn} O, \mathrm{O}^{3}, 2 \overline{\mathrm{K}} \mathrm{Aq}\right) \ldots$ & 28257 & 3 & 9419 \\
\hline$-\left(2 \mathrm{Mn} \mathrm{O}^{5}, 2 \overline{\mathrm{K}} \mathrm{Aq}\right) \ldots \ldots$ & -14765 & 5 & -2953 \\
\hline$-(\mathrm{Mn}, \mathrm{O}) \ldots \ldots \ldots$ & -21511 & 1 & -21511 \\
\hline$-\left(2 \overline{\mathrm{Cr}}, \mathrm{O}^{3}, \mathrm{Aq}\right)$ & -18868 & 3 & -6289 \\
\hline$-\left(\mathrm{JH} \mathrm{Aq}, \mathrm{O}^{3}\right) \ldots \ldots$ & -42542 & 3 & -14181 \\
\hline$-\left(\mathrm{H}^{2} \mathrm{O}, \mathrm{O}, \mathrm{Aq}\right) \ldots \ldots$ & +23074 & 1 & +23074 \\
\hline
\end{tabular}

Mit Rücksicht darauf, wie diese Werthe berechnet sind, verweise ich auf meine Abhandlung in Poggendorff's Annalen.

Universitätslaboratorium zu Kopenhagen, November 1873. 